\title{
Impact of Digit Amputation on Dogs Competing in Agility
}

\author{
Debra C. Sellon ${ }^{1}$ Denis J. Marcellin-Little ${ }^{20}$ Michelle Powers ${ }^{3}$ Sarah Fernandezlopez ${ }^{4}$ \\ Kimberley L. Cullen ${ }^{5}$
}

${ }^{1}$ Department of Veterinary Clinical Sciences, College of Veterinary
Medicine, Washington State University, Pullman, Washington,
United States
${ }^{2}$ Department of Surgical and Radiological Sciences, School of
Veterinary Medicine, University of California, Davis, California,
United States
${ }^{3}$ Boston Mobile Veterinary Specialists, Boston, Massachusetts, United States

VCOT Open 2021;4:e51-e57.
Address for correspondence Debra C. Sellon, DVM, PhD, DACVIM, Department of Veterinary Clinical Sciences, PO Box 646610, College of Veterinary Medicine, Washington State University, Pullman, WA 99164-6610, United States (e-mail: dsellon@wsu.edu).

${ }^{4}$ Bad Dog Agility, Pearland, Texas, United States

5 School of Human Kinetics and Recreation, Memorial University of Newfoundland, St. John's, Newfoundland, Canada

\begin{abstract}
Keywords

- dogs

- digit amputation

- canine agility

- digit injury

Objectives This work sought to determine the prognosis for dogs for return to athletic function in canine agility competitions after digit amputation.

Materials and Methods Data were collected using an Internet-based survey of owners of agility dogs with digit disorders that required amputation. Signalment and physical characteristics were compared between dogs with traumatic and nontraumatic disorders. Athletic performance before and after amputation was assessed subjectively by owner opinion and objectively for eight dogs using competition performance records.

Results Problems leading to digit amputation in 46 dogs included acute or chronic traumatic lesions (30 dogs), infection (7 dogs), neoplasia (7 dogs), and cystic lesions ( 2 dogs). There were no differences in signalment or physical characteristics between dogs with traumatic and nontraumatic disorders. Of the 46 dogs, 39 (84.7\%) had returned to agility at the time of the survey and $94.6 \%$ of the owners (37/39) were satisfied with performance after amputation. No dog failed to return to agility as a result of the digit disorder or amputation. There was no difference in mean competition speed before and after amputation.

Clinical Significance Most agility dogs undergoing digit amputation perform satisfactorily after surgery, although modifications to performance criteria may be appropriate for some.
\end{abstract}

\section{Introduction}

As the popularity of canine agility competitions has increased, there has been a concomitant increased interest in methods to improve diagnosis and treatment of agilityassociated injuries in dogs. Approximately one-third of agil-

received

December 21, 2020

accepted after revision

February 23, 2021
DOI https://doi.org/

$10.1055 / \mathrm{s}-0041-1731436$.

ISSN 2625-2325. ity dogs experience one or more injury during their competitive career with more than a quarter of those dogs having more than one injury. ${ }^{1}$ The most common anatomical sites of injury in agility dogs include shoulders, back, neck, and phalanges. ${ }^{1-3}$ Risk factors for digit injuries in dogs training and competing in agility have been described. ${ }^{4}$ Owners
(C) 2021. The Author(s).

This is an open access article published by Thieme under the terms of the Creative Commons Attribution License, permitting unrestricted use, distribution, and reproduction so long as the original work is properly cited. (https://creativecommons.org/licenses/by/4.0/)

Georg Thieme Verlag KG, Rüdigerstraße 14, 70469 Stuttgart, Germany 
anecdotally report that most dogs with digit injuries return to training and competition at a level comparable to preinjury performance.

Digit amputation is generally considered to result in a good functional outcome for dogs. ${ }^{5,6}$ 'Good functional outcome' is not well-defined and there are no reports of athletic performance measures after digit amputation. We sought to describe factors related to digit amputation in agility dogs and hypothesized that digit amputation is not associated with a decrease in performance as measured by owner perception and mean speed in competition. This hypothesis was tested using a subset of data from a previous retrospective study related to digit injuries in agility dogs, ${ }^{4}$ previously unreported data, and competition records.

\section{Materials and Methods}

\section{Survey Methodology}

A retrospective cross-sectional 91-item survey was developed and distributed by means of a commercial Internet survey site (SurveyMonkey, Palo Alto, CA) as previously described $^{4}$ to facilitate the collection of information on digit disorders in dogs active in agility activities. For the present study, the dataset was examined to identify dogs that had any digit other than the dewclaw on any paw amputated for any reason and for which there was no indication of metacarpal or metatarsal involvement. The survey included sections related to the nature of the digit disorder, physical descriptors of the dog, circumstances of the injury or pathology of the affected digit, agility training and performance characteristics before and after amputation, dog signalment, and handler demographic information. Veterinary records were not obtained to confirm accuracy of owner responses.

Survey respondents were requested to categorize the digit problem as a sprain or strain, fracture, arthritis, broken or ripped nail, dislocation, infection, tumour, or other. Respondents could choose more than one condition if applicable. Responses were subsequently categorized by the investigators as trauma (including sprain or strain, fracture, arthritis, broken or avulsed nail, dislocation), infection, or neoplasia. Respondents identified the affected paw, digit and phalanx, or phalanges involved. Labelled anatomical drawings and drop-down response lists were provided to improve clarity and consistency of the data that were provided. Multiple anatomic areas could be selected. Comment boxes were included so that additional information could be provided where appropriate.

Demographic data included signalment (birth year, sex, breed), body weight, height at the withers, and assessment of body condition. Body weight was classified as underweight, thin, neither thin nor heavy, heavy, or overweight with written descriptions and images provided for each category. Date of amputation was recorded. The age of the dog at the time of surgery was calculated based on birth year and date of surgery.

Handlers were asked to designate the time after surgery at which the dog initially returned to training and returned to competition. They were asked to categorize agility performance after digit amputation as improved, full return to activity, satisfactory return to activity, or unsatisfactory or limited return to activity. Respondents were encouraged to use comment boxes to provide details about performance after amputation. Handlers provided an estimation of training and competition frequency before and after amputation and descriptions of changes in performance criteria.

\section{Performance Records}

Survey respondents provided registration numbers and permission to access performance records from the American Kennel Club (AKC) and United States Dog Agility Association (USDAA). The AKC provided records of performance for each dog and anonymized performance data for all dogs in agility competition in 2012. The USDAA did not provide performance data despite documented owner consent and requests for these data.

In AKC agility, a qualifying run is any run in which the dog completes the course within the maximum allowable time with no performance faults such as incorrect obstacles, refusals, missed contact zones, or dropped bars. The mean speed for all qualifying runs in yards per second (YPS) in regular Excellent/Masters level jumpers with weaves (JWW) and standard (STD) agility courses was calculated for each individual dog that competed in AKC-sanctioned events in 2012. The mean individual dog speeds were used to determine the mean speed of all dogs for each jump height in each type of class. A similar calculation was performed to determine the mean speed of all Border Collie dogs at each jump height in each type of class.

The effects of digit amputation on agility performance were assessed by comparing speed of individual dogs in regular Excellent/Masters level JWW and STD agility courses before and after surgery. Dogs with a minimum of 10 valid YPS data points from qualifying runs in each type of course before and after surgery were included in final analysis. When more than 10 data points from qualifying runs were available, the data from the 10 qualifying runs closest to the date of surgery, excluding the actual month of surgery, were used. Pre-amputation speed, defined as mean speed in 10 qualifying runs immediately preceding the month of surgery, was compared with post-amputation speed, defined as mean speed in 10 qualifying runs immediately after the month of surgery. In addition, for each dog jumping in the 20-inch $(50 \mathrm{~cm})$ regular jump height the mean speed for all lifetime Excellent/Masters level qualifying runs was calculated and compared with the mean speeds of all dogs which competed at the 20-inch regular jump height in 2012. This comparison was repeated for dogs of Border Collie breed.

\section{Statistical Analysis}

Descriptive statistics (mean, standard deviation, median, and range) were calculated for continuous variables. Categorical variables were summarized in contingency tables. Physical characteristics between the group of dogs with amputation secondary to trauma and the group with amputation secondary to nontraumatic disorders were compared by $t$-test for continuous variables (age, body weight, height) and by chi-squared or Fischer exact tests for 
categorical variables (breed, other physical characteristics). Performance speeds in YPS were compared for each individual dog before and after amputation and for amputation dogs as compared with all dogs competing in AKC competitions using a $t$-test, if data were normally distributed (Kolmogorov-Smirnov test), or a Mann-Whitney rank sum test, if not normally distributed. Results of analysis were considered significant if $p<0.05$.

\section{Results}

There were 53 dogs reported to have undergone digit amputation. Seven dogs were excluded from the final dataset. Four dogs were excluded because the first digit (dewclaw) was amputated, one dog was a duplicate entry, one respondent provided inconsistent data and, one dog had reported metacarpal/metatarsal involvement. The final dataset included 46 dogs with digit amputations due to trauma (30 dogs), infections ( 7 dogs), neoplastic conditions ( $7 \mathrm{dogs}$ ), or cysts (2 dogs). Of the dogs with trauma, the specific injuries described included fracture (15 dogs), torn ligament or tendon (5 dogs), dislocation ( 5 dogs), sprain or strain (3 dogs), broken or ripped nail (2 dogs), arthritis ( $1 \mathrm{dog})$, and puncture wound (1 dog).

The distribution of affected digits is shown in - Table 1. In dogs with traumatic lesions that led to amputation, the fifth digit was most commonly affected (16/30 digits, 53.3\%). The fourth digit was most commonly affected in dogs with nontraumatic disorders $(6 / 16,37.5 \%)$. The distal phalanx was affected in 21 dogs, the middle and distal phalanges in 6 dogs, the middle phalanx in 6 dogs, the proximal and middle phalanges in 3 dogs, the proximal phalanges in 5 dogs, and all phalanges in 2 dogs. Three respondents were unsure of which phalanges were affected.
The mean age of all dogs at the time of amputation was $5.8 \pm 3.2$ years with a median of 6 years (range $<1$ to 12 years). The mean age of dogs that underwent amputation because of a traumatic injury was $5.4 \pm 3.2$ years with a median age of 5 years. The mean age of dogs with nontraumatic disorders leading to amputation was $6.8 \pm 2.9$ years with a median of 7.0 years. There was no significant difference in age between dogs with traumatic lesions and nontraumatic lesions.

The most common breeds were Border Collie (10 dogs), mixed breed ( 5 dogs), Australian Shepherd (4 dogs), Labrador Retriever (3 dogs), and Whippet (3 dogs). There were 2 dogs each of Golden Retriever, Miniature Schnauzer, Vizsla, or Shetland Sheepdog breed. There was one each of Belgian Malinois, Briard, Doberman Pinscher, Gordon Setter, Irish Water Spaniel, Kromfohrländer, Miniature American Shepherd, Parson Russell Terrier, Pembroke Welsh Corgi, Pharaoh Hound, Rat Terrier, Weimaraner, and unlisted breed.

Height at the withers, body weight, and weight to height ratio for all dogs undergoing digit amputation are shown in -Table 2. Other physical characteristics, including presence of dewclaws, nail length, and paw fur length are described in - Table 3. There were no significant differences in physical characteristics between dogs with amputation for traumatic injuries or other types of disorders.

Of the 46 dogs with digit amputations, 39 returned to agility training and competition (84.7\%), 2 dogs returned to training only, 4 dogs were still in recovery at the time of the survey responses, and $1 \mathrm{dog}$ did not return to agility. The dog that did not return to agility was retired from the sport for reasons unrelated to agility. One of the two dogs that returned to training only was reported to have a torn cranial cruciate ligament that impacted return to competition and one was not yet old enough to compete. No dogs were

Table 1 Frequency of specific digit amputation in dogs with amputations secondary to traumatic lesions, dogs with amputations secondary to nontraumatic lesions, and all dogs

\begin{tabular}{|c|c|c|c|c|c|c|}
\hline Group & Foot & Digit 2 & Digit 3 & Digit 4 & Digit 5 & Total \\
\hline \multirow[t]{5}{*}{ Dogs with traumatic lesions $(n=30)$} & Left front & 1 & 2 & 1 & 5 & $9(30.0 \%)$ \\
\hline & Right front & 3 & 1 & 1 & 5 & $10(33.3 \%)$ \\
\hline & Left rear & 1 & 0 & 2 & 6 & $9(30.0 \%)$ \\
\hline & Right rear & 0 & 1 & 1 & 0 & $2(6.7 \%)$ \\
\hline & TOTAL & $5(16.7 \%)$ & $4(13.3 \%)$ & $516.7 \%)$ & $16(53.3 \%)$ & 30 \\
\hline \multirow[t]{5}{*}{ Dogs with nontraumatic lesions $(n=16)$} & Left front & 1 & 2 & 2 & 1 & $6(37.5 \%)$ \\
\hline & Right front & 3 & 1 & 3 & 0 & 7 (43.8\%) \\
\hline & Left rear & 0 & 1 & 1 & 1 & $3(18.8 \%)$ \\
\hline & Right rear & 0 & 0 & 0 & 0 & $0(0.0 \%)$ \\
\hline & TOTAL & $4(25.0 \%)$ & $4(25.0 \%)$ & $6(37.5 \%)$ & $2(12.5 \%)$ & 16 \\
\hline \multirow[t]{5}{*}{ All dogs $(n=46)$} & Left front & 2 & 4 & 3 & 6 & $15(32.6 \%)$ \\
\hline & Right front & 6 & 2 & 4 & 5 & $17(36.9 \%)$ \\
\hline & Left rear & 1 & 1 & 3 & 7 & $12(26.1 \%)$ \\
\hline & Right rear & 0 & 1 & 1 & 0 & $2(4.3 \%)$ \\
\hline & TOTAL & $9(19.6 \%)$ & $8(17.4 \%)$ & $11(23.9 \%)$ & $18(39.1 \%)$ & 46 \\
\hline
\end{tabular}


e54 Digit Amputation in Agility Dogs Sellon et al.

Table 2 Height, body weight, and weight to height ratio for dogs with digit amputation secondary to an acute or chronic traumatic lesion $(n=30)$, nontraumatic lesions $(n=16)$, and all dogs with digit amputation $(n=46)$

\begin{tabular}{|l|l|l|l|l|l|}
\hline Variable & Group & Mean \pm SD & Median & Minimum & Maximum \\
\hline Height at withers $(\mathrm{cm})$ & Trauma & $48.8 \pm 10.1$ & 49.5 & 25.4 & 64.8 \\
\cline { 2 - 6 } & No trauma & $52.8 \pm 7.9$ & 52.8 & 38.7 & 66.0 \\
\cline { 2 - 6 } & All dogs & $50.3 \pm 9.4$ & 50.8 & 25.4 & 66.0 \\
\hline \multirow{5}{*}{ Body weight $(\mathrm{kg})$} & Trauma & $16.9 \pm 6.2$ & 15.9 & 5.4 & 28.6 \\
\cline { 2 - 6 } & No trauma & $20.5 \pm 8.0$ & 21.1 & 9.1 & 35.4 \\
\cline { 2 - 6 } & All dogs & $18.2 \pm 7.0$ & 16.4 & 5.5 & 35.5 \\
\hline \multirow{3}{*}{ Weight: height ratio } & Trauma & $1.90 \pm 0.47$ & 1.84 & 0.91 & 3.33 \\
\cline { 2 - 6 } & No trauma & $2.11 \pm 0.60$ & 2.14 & 1.27 & 3.06 \\
\cline { 2 - 6 } & All dogs & $1.97 \pm 0.52$ & 1.87 & 0.91 & 3.33 \\
\hline
\end{tabular}

Abbreviation: SD, standard deviation.

Note: There were no significant differences in any variable between dogs with traumatic lesions and dogs with nontraumatic lesions.

Table 3 Physical characteristics of dogs with digit amputation

\begin{tabular}{|l|l|l|l|l|}
\hline Variable & Classification & $\begin{array}{l}\text { Traumatic lesions } \\
(\boldsymbol{n}=\mathbf{3 0})\end{array}$ & $\begin{array}{l}\text { Nontraumatic lesions } \\
(\boldsymbol{n}=\mathbf{1 6})\end{array}$ & $\begin{array}{l}\text { All dogs } \\
(\boldsymbol{n}=\mathbf{4 6})\end{array}$ \\
\hline \multirow{5}{*}{ Gender } & Female, all & $19(63.3 \%)$ & $7(43.8 \%)$ & $26(56.5 \%)$ \\
\cline { 2 - 5 } & Female, intact & 3 & 1 & 4 \\
\cline { 2 - 5 } & Female, altered & 16 & 5 & 21 \\
\cline { 2 - 5 } & Female, altered, hormone-sparing & 0 & 1 & 1 \\
\cline { 2 - 5 } & Male, all & $11(36.7 \%)$ & $9(56.3 \%)$ & $20(43.5 \%)$ \\
\cline { 2 - 5 } & Male, intact & 1 & 2 & 3 \\
\cline { 2 - 5 } & Male, altered & 10 & 7 & 17 \\
\cline { 2 - 5 } & Male, altered, hormone-sparing & 0 & 0 & 0 \\
\hline Front dew claws & Present & $14(46.7 \%)$ & $10(62.5 \%)$ & $24(52.2 \%)$ \\
\cline { 2 - 5 } & Absent & $16(53.3 \%)$ & $6(37.5 \%)$ & $22(47.8 \%)$ \\
\hline Rear dew claws & Present & $0(0.0 \%)$ & $1(6.3 \%)$ & $1(2.2 \%)$ \\
\cline { 2 - 5 } & Absent & $30(100.0 \%)$ & $15(93.8 \%)$ & $45(97.8 \%)$ \\
\hline \multirow{5}{*}{ Nail length } & Long & $2(6.7 \%)$ & $0(0.0 \%)$ & $2(4.3 \%)$ \\
\cline { 2 - 5 } & Medium & $23(76.7 \%)$ & $9(56.3 \%)$ & $32(69.6 \%)$ \\
\cline { 2 - 5 } & Short & $5(16.7 \%)$ & $7(43.8 \%)$ & $12(26.1 \%)$ \\
\hline \multirow{5}{*}{ Paw fur } & Long & $1(3.3 \%)$ & $0(0.0 \%)$ & $1(2.2 \%)$ \\
\cline { 2 - 5 } & Medium & $7(23.3 \%)$ & $7(43.8 \%)$ & $31(30.4 \%)$ \\
\cline { 2 - 5 } & Short, naturally or trimmed & $22(73.3 \%)$ & $9(56.3 \%)$ & $31.4 \%)$ \\
\hline
\end{tabular}

Note: There were no significant differences in any variable between dogs with traumatic lesions and dogs with nontraumatic lesions.

reported to have failed to return to agility competition because of digit amputation.

The average time from amputation until return to agility training was $11.6 \pm 8.1$ weeks. Median time until return to training was 9.0 weeks with a range of 2 to 44 weeks. Respondents reported changes-to-performance criteria as a result of the surgery were reported for 14 of 39 dogs (35.9\%). Of these, three respondents indicated that the dog was jumping a lower jump height, four altered the dog's performance criteria on contact obstacles (A-frame, dogwalk, teeter), and others indicated that they made changes to handling in an attempt to promote changes in the dog's performance (e.g. slower, wider turns, more cautious).

Athletic performance after surgery was characterized as improved $(8 / 39,20.5 \%)$, full return to previous level of performance $(16 / 39,41.0 \%)$, satisfactory $(13 / 39,33.3 \%)$, moderate return to performance $(1 / 39,2.6 \%)$, and unsatisfactory or limited return to performance (1/39, 2.6\%).

Performance records were available for 23 dogs with digit amputations (17 dogs with amputations due to trauma, 4 with neoplastic conditions, and 2 with infections). There were 8 dogs with a minimum of 10 qualifying runs in 


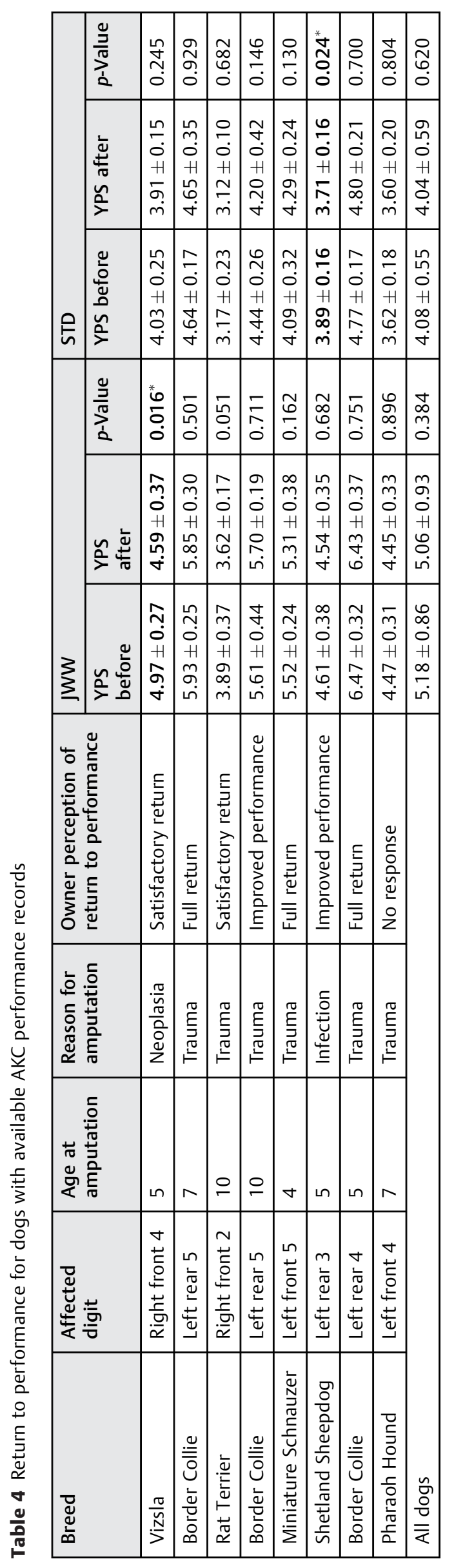

Excellent/Masters level JWW and STD courses prior to amputation and after amputation. Pre- and postamputation speed are shown in - Table 4 .

The mean speed of all dogs and all Border Collies competing at the 20-inch jump height in 2012 was compared with the mean pre-amputation speed of dogs that underwent surgery ( - Table 5 ). The data from 2012 were used because it was the mean year in which injury occurred in the previous study of digit injuries in dogs. ${ }^{4}$ Mean pre-amputation speed for JWW competitions was faster for dogs with digit amputations than for all dogs at that jump height $(p=0.021)$ but there was no difference when comparing the speed of affected Border Collies to all Border Collies in competition.

\section{Discussion}

Risk factors for digit injuries in agility dogs include absence of dewclaws, Border Collie breed, long nails, and greater weight-to-height ratio. ${ }^{4}$ Most affected dogs return to athletic performance after conservative therapy. Digit amputation in dogs is generally considered to have a good prognosis based on owner-reported satisfaction with the procedure..$^{5-11}$ Previous reports do not include measures of athletic function after digit amputation. This study sought to more closely examine return to athletic performance in agility competitions after amputation of a digit.

Of the 46 dogs with digit amputations, surgery was performed to address traumatic lesions in the majority (30/46, 65\%). The percentage of dogs with traumatic injuries is larger than in a previous retrospective study of canine digit amputations, in which $11 / 35$ (31.4\%) had traumatic lesions. ${ }^{5}$ The increased prevalence of trauma is expected in a population of agility dogs as is the proportion of Border Collies (10/46 dogs, $21.7 \%$ ) as compared with the study by Kaufman (1/33 dogs, 3.0\%). ${ }^{5}$ The preponderance of traumatic lesions and Border Collies in this study may be responsible for the apparent differences in age and body weight as compared with Kaufman's report. In the agility dogs, the mean age at the time of amputation was 5.8 years as compared with 7.8 years in Kaufman's report. The mean body weight for affected agility dogs was $18.2 \mathrm{~kg}$ as compared with $30.1 \mathrm{~kg}$ for Kaufman's dogs.

The distribution of affected digits in this study ( - Table $\mathbf{1}$ ) differed from the distribution observed by Kaufman in which the frequency of amputation was $7 / 32$ (21.9\%) for digit 2, 9/32 (28.1\%) for digit 3, 8/32 (22.9\%) for digit 4 , and $5 / 33(14.3 \%)$ for digit 5 . The apparent over-representation of digit 5 amputations among agility dogs was consistent with the previous report of a large number of injuries to that digit in this population. ${ }^{4}$ Most of the digit 5 amputations $(16 / 18,88.9 \%)$ were related to trauma as compared with $14 / 28(50.0 \%)$ of amputations for digits 2,3 , and 4 , suggesting a high likelihood of trauma to the fifth digit in agility dogs. Differences in prognosis for return to performance related to the specific toe that was amputated were inapparent, but numbers were small and such a difference cannot be ruled out on the basis of currently available information. 
Table 5 Mean speed in YPS of dogs in the 20-inch jump height in regular JWW and STD courses

\begin{tabular}{|l|l|l|l|l|l|}
\hline \multicolumn{2}{|c|}{} & \multicolumn{2}{|l|}{ All AKC dogs at $\mathbf{2 0}$ inches } & \multicolumn{2}{l|}{ Border Collies at 20 inches } \\
\cline { 3 - 7 } \multicolumn{2}{|c|}{} & $\begin{array}{l}\text { Dogs with digit } \\
\text { amputations }\end{array}$ & $\begin{array}{l}\text { All AKC dogs } \\
\text { of any breed }\end{array}$ & $\begin{array}{l}\text { Dogs with digit } \\
\text { amputations }\end{array}$ & $\begin{array}{l}\text { All AKC } \\
\text { Border Collies }\end{array}$ \\
\hline \multirow{2}{*}{ JWW } & Mean \pm SD YPS & $5.53 \pm \mathbf{0 . 7 8 ^ { * }}$ & $4.94 \pm \mathbf{0 . 7 6}$ & $5.69 \pm 0.68$ & $5.48 \pm 0.68$ \\
\cline { 2 - 7 } & Number of dogs & 8 & 4,042 & 6 & 4,469 \\
\hline \multirow{2}{*}{ STD } & Mean \pm SD YPS & $4.24 \pm 0.57$ & $3.89 \pm 0.56$ & $4.32 \pm 0.44$ & $4.26 \pm 0.51$ \\
\cline { 2 - 7 } & Number of dogs & 8 & 3,860 & 6 & 1,435 \\
\hline
\end{tabular}

Abbreviations: AKC, American Kennel Club; JWW, jumpers with weaves course; STD, standard course; YPS, yards per second.

Note: The mean speed of dogs with amputations is calculated based on mean speed for all qualifying runs at that height in the specified class at AKCsanctioned events through the lifetime of the dog. The speed for all AKC dogs of any breed and all Border Collies was calculated as the average of the means for each individual dog based on qualifying runs in the specified class in the year 2012. Bold and ${ }^{*}=p<0.05$. See text for additional information.

According to owners, no dogs failed to return to agility because of digit amputation. Owners subjectively characterized performance after amputation as 'returned to normal' or 'improved' in $61.5 \%$ of dogs $(24 / 39)$ and 'satisfactory' in another $33.3 \%$ of dogs (13/39). We cannot rule out the possibility of a caregiver placebo effect ${ }^{12}$ which may have resulted in more positive owner perceptions of postamputation performance than might have been provided by an unbiased observer. Several owners who indicated satisfactory return to performance included comments suggestive of ongoing intermittent lameness after exercise, increased turning radius, and similar performance concerns. The overall subjective assessment of return to athletic performance in most dogs was supported by objective comparison of speed in competition before and after surgery. Only two of eight dogs (25\%) for which competition records were available showed decreased speed in competition. Each of these dogs had a small decrease in average speed in one of the two course types which were assessed (-Table 4 ).

Digit injuries in agility dogs have been suggested to be related to speed, with more digit injuries occurring in faster dogs such as Border Collies. The mean JWW speed for dogs with amputations was faster than for all dogs competing at the same jump height. This difference disappears when only Border Collie speeds are compared, making it difficult to determine whether speed alone is a risk factor or there is some characteristic of Border Collies that might render them more susceptible to an injury requiring amputation.

This study has several potential limitations, the most significant of which is that most data were provided by owners and not via review of veterinary records. The strengths and limitations of this type of survey data were recently reviewed by Sellon and colleagues. ${ }^{4}$ Limitations include potential bias in respondents and inaccurate recall of events by owners. Performance data provided by AKC strengthened the ability to assess effects of digit amputation on performance but were available for only eight dogs. Objective performance data are critical to analysis of health effects on competition dogs and should be used in the same way that racing records are used to enhance diagnosis and treatment of equine musculoskeletal injuries. Numerous factors unrelated to digit injury including age, experience, change in training or performance criteria, or handler errors on individual runs could have influenced competition speed in individual dogs in this study. If data were available for more dogs, unrelated factors would be expected to be less impactful on conclusions.

Data presented in this manuscript are consistent with previous reports suggesting that digit amputation is welltolerated in dogs. Those conclusions are extended with subjective and objective evidence that dogs undergoing digit amputations are very likely to return to satisfactory or better performance, although some modification to performance criteria may be appropriate for individual dogs.

\section{Author Contributions}

We certify that all authors meet the qualifications for authorship as listed below: DS, DM-L, MP, and SF substantially contributed to the conception or design of the work. DS acquired data. DS, DM-L, and SF analysed data. DS, DM-L, SF, MP, and KC interpreted data for the work. DS and DM-L did initial drafting of the work and revised it critically for important intellectual content. All authors agreed to be accountable for all aspects of the work in ensuring that questions related to the accuracy or integrity of any part of the work are appropriately investigated and resolved. All authors contributed to the article and approved the submitted version.

\section{Funding}

This research was suppored in part by funds from Washington State University.

\section{Conflict of Interest}

The authors declare that the research was conducted in the absence of any commercial or financial relationships that could be construed as a potential conflict of interest.

\section{Acknowledgments}

The authors thank Katherine Martucci for assistance with survey preparation and data collection.

\section{References}

1 Cullen KL, Dickey JP, Bent LR, Thomason JJ, Moëns NM. Internetbased survey of the nature and perceived causes of injury to dogs participating in agility training and competition events. J Am Vet Med Assoc 2013;243(07):1010-1018 
2 Kerr ZY, Fields S, Comstock RD. Epidemiology of injury among handlers and dogs competing in the sport of agility. J Phys Act Health 2014;11(05):1032-1040

3 Levy M, Hall C, Trentacosta N, Percival M. A preliminary retrospective survey of injuries occurring in dogs participating in canine agility. Vet Comp Orthop Traumatol 2009;22(04): 321-324

4 Sellon DC, Martucci K, Wenz JR, Marcellin-Little DJ, Powers M, Cullen KL. A survey of risk factors for digit injuries among dogs training and competing in agility events. J Am Vet Med Assoc 2018;252(01):75-83

5 Kaufman KL, Mann FA. Short- and long-term outcomes after digit amputation in dogs: 33 cases (1999-2011). J Am Vet Med Assoc 2013;242(09):1249-1254

6 Pearson EL, Whelan M, Bracker K. Escalator-related injuries in 30 dogs (2007-2014). J Vet Emerg Crit Care (San Antonio) 2017;27(04): 434-438
7 Henry CJ, Brewer WG Jr, Whitley EM, et al; Veterinary Cooperative Oncology Group (VCOG) Canine digital tumors: a veterinary cooperative oncology group retrospective study of 64 dogs. J Vet Intern Med 2005;19(05):720-724

8 Hickman J. Greyhound injuries. J Small Anim Pract 1975;16(08): 455-460

9 Liptak JM, Dernell WS, Rizzo SA, Withrow SJ. Partial foot amputation in 11 dogs. J Am Anim Hosp Assoc 2005;41(01):47-55

10 Muir P, Pead MJ. Chronic lameness after digit amputation in three dogs. Vet Rec 1998;143(16):449-450

11 Wobeser BK, Kidney BA, Powers BE, et al. Diagnoses and clinical outcomes associated with surgically amputated canine digits submitted to multiple veterinary diagnostic laboratories. Vet Pathol 2007;44(03):355-361

12 Conzemius MG, Evans RB. Caregiver placebo effect for dogs with lameness from osteoarthritis. J Am Vet Med Assoc 2012;241(10): $1314-1319$ 